\title{
Corticospinal excitability remains unchanged in the presence of residual force enhancement and does not contribute to increased torque production
}

\author{
Jasmin Frischholz ${ }^{1}$, Brent J. Raiteri ${ }^{1}$, Andrew G. Cresswell ${ }^{2}$, Daniel Hahn ${ }^{\text {Corresp. } 1,2}$ \\ ${ }^{1}$ Human Movement Science, Faculty of Sport Science, Ruhr University Bochum, Bochum, Germany \\ 2 School of Human Movement and Nutrition Sciences, University of Queensland, Brisbane, Australia \\ Corresponding Author: Daniel Hahn \\ Email address: daniel.hahn@rub.de
}

Background. Following stretch of an active muscle, muscle force is enhanced, which is known as residual force enhancement ( $\mathrm{rFE}$ ). As earlier studies found apparent corticospinal excitability modulations in the presence of $\mathrm{rFE}$, this study aimed to test whether corticospinal excitability modulations contribute to rFE.

Methods. Fourteen participants performed submaximal plantar flexion stretch-hold and fixed-end contractions at $30 \%$ of their maximal voluntary soleus muscle activity in a dynamometer. During the steady state of the contractions, participants either received subthreshold or suprathreshold transcranial magnetic stimulation (TMS) of their motor cortex, while triceps surae muscle responses to stimulation were obtained via electromyography (EMG), and net ankle joint torque was recorded. B-mode ultrasound imaging was used to confirm muscle fascicle stretch during stretch-hold contractions in a subset of participants.

Results. Following stretch of the plantar flexors, an average rFE of $7 \%$ and $11 \%$ was observed for contractions with subthreshold and suprathreshold TMS, respectively. 41-46 ms following subthreshold TMS, triceps surae muscle activity was suppressed by $19-25 \%$, but suppression was not significantly different between stretch-hold and fixed-end contractions. Similarly, the reduction in plantar flexion torque following subthreshold TMS was not significantly different between contraction conditions. Motor evoked potentials, silent periods and superimposed twitches following suprathreshold TMS were also not significantly different between contraction conditions.

Discussion. As TMS of the motor cortex did not result in any differences between stretch-hold and fixedend contractions, we conclude that rFE is not linked to changes in corticospinal excitability. 


\section{Corticospinal excitability remains unchanged in the}

3 presence of residual force enhancement and does not

4 contribute to increased torque production

5

6

7

8

9

10 11

12

13

14

15
Jasmin Frischholz ${ }^{1}$, Brent J. Raiteri ${ }^{1}$, Andrew G. Cresswell ${ }^{2}$, Daniel Hahn ${ }^{1,2}$

${ }^{1}$ Human Movement Science, Faculty of Sport Science, Ruhr University Bochum, Germany

${ }^{2}$ School of Human Movement and Nutrition Sciences, University of Queensland, Brisbane, Australia

Corresponding Author:

Daniel Hahn

Human Movement Science, Faculty of Sport Science, Ruhr University Bochum,

Gesundheitscampus Nord 10, 44801 Bochum, Germany

Email address: daniel.hahn@rub.de 


\section{Abstract}

21 Background. Following stretch of an active muscle, muscle force is enhanced, which is known as residual force enhancement $(\mathrm{rFE})$. As earlier studies found apparent corticospinal excitability modulations in the presence of $\mathrm{rFE}$, this study aimed to test whether corticospinal excitability modulations contribute to rFE.

25 Methods. Fourteen participants performed submaximal plantar flexion stretch-hold and fixed26 end contractions at $30 \%$ of their maximal voluntary soleus muscle activity in a dynamometer. 27 During the steady state of the contractions, participants either received subthreshold or suprathreshold transcranial magnetic stimulation (TMS) of their motor cortex, while triceps surae muscle responses to stimulation were obtained via electromyography (EMG), and net ankle joint torque was recorded. B-mode ultrasound imaging was used to confirm muscle fascicle stretch during stretch-hold contractions in a subset of participants.

Results. Following stretch of the plantar flexors, an average rFE of $7 \%$ and $11 \%$ was observed for contractions with subthreshold and suprathreshold TMS, respectively. 41-46 ms following subthreshold TMS, triceps surae muscle activity was suppressed by $19-25 \%$, but suppression was not significantly different between stretch-hold and fixed-end contractions. Similarly, the reduction in plantar flexion torque following subthreshold TMS was not significantly different between contraction conditions. Motor evoked potentials, silent periods and superimposed twitches following suprathreshold TMS were also not significantly different between contraction conditions.

Discussion. As TMS of the motor cortex did not result in any differences between stretch-hold and fixed-end contractions, we conclude that $\mathrm{rFE}$ is not linked to changes in corticospinal excitability. 


\section{Introduction}

45 It is well known that stretch of an active muscle results in increased force production during the

46

47

48

49

50

51

52

53

54

55

56

57

58

59

60

61

62

63

64

65

66

67

68

69

70

71

72

73

74

75

76

77

78

79

80

81

82

83 isometric steady state following stretch compared with the steady-state force produced at the same muscle length and activation level during a fixed-end contraction. This is referred to as residual force enhancement ( $\mathrm{rFE}$ ), which was initially investigated in situ (Abbott \& Aubert, 1952). Decades later, rFE was investigated in isolated muscle fibres and two opposing mechanisms were suggested: (1) that $\mathrm{rFE}$ following active muscle stretch results from nonuniformities in sarcomere lengths (Julian \& Morgan, 1979), and (2) that rFE is due to the engagement of a parallel non-contractile element during active stretch (Edman, Elzinga \& Noble, 1978). Since then, numerous studies have investigated the development of rFE and suggested additional potential underlying mechanisms. These suggestions include stretch-induced increases in the number of cross-bridge attachments and/or the attachment of the second myosin head, and an increase in the average cross-bridge force and strain (Brunello et al., 2007; Rassier, 2012; Herzog, 2014). Lately, the engagement of a parallel non-contractile element during active stretch has been related to titin, which might increase its force contribution in the presence of calcium and by interacting with actin (Nishikawa, 2016; Herzog, 2018; Freundt \& Linke, 2019).

Complementary to in vitro research, several in vivo studies have investigated rFE (as approximated by net joint torque) during electrically-stimulated and voluntary contractions (Seiberl, Power \& Hahn, 2015; Chapman et al., 2018). rFE during voluntary contractions has been observed for small muscles of the thumb (Lee and Herzog, 2002), for large muscles of the lower limb (Pinniger \& Cresswell, 2007; Seiberl et al., 2010), and during multi-joint multimuscle contractions of the lower limbs (Hahn et al., 2010). The potential relevance of rFE during human movement was demonstrated in studies that investigated rFE during submaximal voluntary contractions and at joint angle configurations that mimicked those of human locomotion (Hahn et al., 2010; Seiberl et al., 2013; Paternoster et al., 2016).

Besides $\mathrm{rFE}$, several in vivo studies have documented an activation reduction (AR) during the steady state following active muscle stretch. In contrast to rFE, which is observed when muscle activity is matched between stretch-hold (STR) and fixed-end reference (REF) contractions, AR occurs when force or torque is matched between contraction conditions. AR refers to a reduced muscle activity level needed to maintain a given force/torque following active muscle stretch compared with fixed-end contractions at the same final muscle length. Several studies have reported AR and concluded that neuromuscular efficiency following active muscle stretch is increased, which might be explained by enhanced passive forces due to increased titin forces (Oskouei \& Herzog, 2005; Altenburg et al., 2008; Seiberl et al., 2012; Joumaa \& Herzog, 2013; Jones, Power \& Herzog, 2016; Mazara et al., 2018; Paquin \& Power, 2018). However, Paquin $\&$ Power (2018) also found a rightward shift in the EMG-torque relationship following active stretch compared with fixed-end reference conditions, which indicates that the neuromuscular activation strategy might be altered in the presence of $\mathrm{rFE}$.

Peer) reviewing PDF | (2021:04:59848:2:0:NEW 17 Nov 2021) 
84

85

86

87

88

89

90

91

92

93

94

95

96

97

98

99

100

101

102

103

104

105

106

107

108

109

110

111

112

113

114

115

116

117

118

119

120

121

122

123

Changes in EMG following active stretch have motivated a number of studies to investigate the neural control and/or neural modulations that occur during the isometric steady state following active muscle stretch (Altenburg et al., 2008; Hahn et al., 2012; Paquin \& Power, 2018; Sypkes et al., 2018; Contento, Dalton \& Power, 2019; Jakobi et al., 2020). For example, Altenburg et al. (2008) examined single motor unit behaviour of the vastus lateralis muscle during AR following active muscle stretch. These authors found similar discharge rates of VL motor units between STR and REF conditions, which led them to conclude that a derecruitment of motor units might have occurred during AR (Altenburg et al. 2008).

Two further studies used motor evoked potentials (MEPs) and cervicomedullary motor evoked potentials (CMEPs) to investigate excitability modulations at cortical and spinal sites in the presence of rFE (Hahn et al., 2012; Sypkes et al., 2018). In the first study, Hahn et al. (2012) found larger MEPs and larger V-waves, but unchanged CMEPs, following stretch of the plantar flexors during maximal voluntary contractions. Based on the larger MEPs and unchanged CMEPs, all normalised to Mmax, it was interpreted that cortical excitability increased, but spinal excitability was unchanged in the rFE state. The increased V-waves were considered to represent greater cortical motoneuronal output and/or an increase in spinal stretch reflex excitability following active muscle stretch (Hahn et al. (2012). In the second study, Sypkes et al. (2018) found smaller CMEPs (normalised to Mmax) and an unchanged MEP/CMEP ratio in the presence of $\mathrm{rFE}$ during submaximal dorsiflexion contractions, which was interpreted as reduced spinal excitability, but no change in cortical excitability. However, because it is not reported whether the MEP/Mmax ratio changed or remained constant, and Mmax varied within participants from -20 to $17 \%$ between the ISO and RFE conditions in that study, it is virtually impossible to interpret the provided MEP/CMEP ratio (see supplementary material Table 1 for a detailed explanation). Further, assuming that the MEPs' corresponding Mmax was unchanged, the combined results of the reduced CMEPs and unchanged MEPs in the study of Sypkes et al. (2018) can also be interpreted as reduced spinal excitability and increased cortical excitability (Martin, Gandevia \& Taylor, 2006; Martin et al., 2009). Accordingly, both studies on neural excitability (Hahn et al. 2012 and Sypkes et al. 2018) might indicate increased cortical excitability in the presence of $\mathrm{rFE}$, but this currently remains a matter of debate.

\section{Despite different muscle groups being tested under different levels of voluntary effort, the} ambiguous results from the earlier studies likely rises due to both studies being underpowered and the statistical significance occurring due to chance. That is, the observed changes in CMEPs (Sykpes et al. 2018) and MEPs (Hahn et al. 2012) in the rFE state, while significant, were not large (Cohen's $d_{\mathrm{z}}=0.70$ and 0.75 , respectively). A paired t-test with 11 participants, which was the maximum sample size tested across these studies, would not have been able to reliably detect effects smaller than a Cohen's $d_{z}$ of 0.94 with $80 \%$ power at a two-tailed alpha level of 0.05 . Further, only a few responses (i.e. MEPs) to transcranial magnetic stimulation (TMS) were

Peer) reviewing PDF | (2021:04:59848:2:0:NEW 17 Nov 2021) 
124 averaged ( $\mathrm{n}=6$ in Hahn et al. 2012 and $\mathrm{n}=4$ minus outliers in Sypkes et al. 2018), although it

125

126

127

128

129

130

131

132

133

134

135

136

137

138

139

140

141

142

143

144

145

146

147

148

149

150

151

152

153

154

155

156

157

158

159

160

161

162

163

164 has been shown that as many as 20 MEPs are needed to accurately estimate corticospinal excitability (Brownstein et al., 2018).

Therefore, the aim of this study was twofold. First, we wanted to partly replicate the abovementioned studies and investigate whether corticospinal excitability was altered in the presence of rFE. This was done by eliciting 20 MEPs via TMS in the presence (STR) and absence (REF) of rFE in an adequately-powered study. Second, this study was designed to investigate whether cortical inhibition induced by subthreshold TMS affects force production in the presence of $\mathrm{rFE}$ differently to fixed-end reference contractions without $\mathrm{rFE}$.

Based on a critical evaluation of previous research (Hahn et al., 2012; Sypkes et al., 2018), we predicted that the activation of cortical interneurons and pyramidal neurons by suprathreshold TMS (Rothwell, 1997; Rossini et al., 2015) would result in similar MEPs and superimposed twitches in the presence of rFE compared with the fixed-end reference contractions with matched background muscle activity. Further, we predicted that inhibiting motor cortical neurons by subthreshold TMS (Davey et al., 1994; Petersen et al., 2001; Zuur et al., 2010) would lead to a similar suppression in muscle activity (EMG) and plantar flexion torque in the presence of $\mathrm{rFE}$ compared with fixed-end reference contractions with matched background muscle activity. If both predictions hold, the results would indicate that corticospinal excitability is not altered and does not contribute to the increased torque production in the presence of $\mathrm{rFE}$.

\section{Materials \& Methods}

\section{Participants}

Fourteen recreationally-active participants (six women, $26.7 \pm 5.3$ yrs., $1.77 \pm 0.11 \mathrm{~m}$, and $74.0 \pm$ $16.8 \mathrm{~kg}$ ) voluntarily participated in this study after providing free written informed consent. A total sample size of 10 was calculated to have over $90 \%$ power to detect a minimum effect size of 0.62 with a two-tailed alpha level of 0.05 . This was calculated with Superpower (https://shiny.ieis.tue.nl/anova_power/) from 2000 simulations using data from (Hahn et al., 2012), which incorporated a $2 \times 2$ repeated-measures design and observed a common standard deviation of 5.11 (note a conservative within-subjects factor correlation of 0.5 was used in the simulations). Participants were free of any neuromuscular disorders and injuries to their right lower limb. The experimental protocol was approved by the local Ethics Committee of the Faculty of Sport Science at Ruhr University Bochum, Germany (EKS10072018).

\section{Experimental setup}

During the experiment, participants laid prone on a dynamometer bench with their upper body supported by their forearms and their right foot tightly strapped onto the footplate attachment of a dynamometer (IsoMed 2000, D\&R Ferstl GmbH, GER). The axes of rotation of the ankle joint and the dynamometer were aligned prior to testing and the foot was firmly secured to the footplate using three straps (forefoot, ankle, heel) to minimize heel lift during the plantar flexion

Peer] reviewing PDF | (2021:04:59848:2:0:NEW 17 Nov 2021) 
165

166

167

168

169

170

171

172

173

174

175

176

177

178

179

180

181

182

183

184

185

186

187

188

189

190

191

192

193

194

195

196

197

198

199

200

201

202

203

204

205

contractions. Additionally, the participants' hips were secured to the dynamometer bench with a belt. A computer monitor positioned directly in front of the participants was used to provide visual feedback of their soleus muscle activity (moving 0.25 -s root-mean-square amplitude calculation) during the plantar flexion contractions. Net ankle joint torques and ankle joint angles of the right leg were sampled at $1 \mathrm{kHz}$ using a 16-bit Power-3 1401 and Spike2 data collection system (v.8.01, Cambridge Electronics Design, Cambridge, UK)

\section{Surface electromyography}

Muscle activity and responses to TMS from soleus (SOL), medial gastrocnemius (MG) and lateral gastrocnemius (LG) muscles were recorded via bipolar surface electromyography (EMG). EMG of the antagonistic tibialis anterior (TA) was not obtained as it has been shown that TA EMG during fixed-end plantar flexion is more likely from crosstalk than coactivation (Raiteri, Cresswell \& Lichtwark, 2015). After skin preparation, self-adhesive surface electrodes (Ag/ $\mathrm{AgCl}$, Kendall, ECG Electrodes, $8 \mathrm{~mm}$ recording diameter) were positioned with an interelectrode distance of $20 \mathrm{~mm}$ over the triceps surae muscle bellies following the recommendations of SENIAM (Hermens et al., 1999). A reference electrode was placed over the right fibular head. Cables were fixed to the skin with tape to prevent motion artefacts. EMG signals were amplified 1000 times by a multichannel analogue amplifier (AnEMG12, Bioelectronica, Turin, IT) and band-pass filtered between $10 \mathrm{~Hz}$ and $4.4 \mathrm{kHz}$, prior to being sampled at $5 \mathrm{kHz}$. EMG and torque/angle data were synchronised and sampled using the same analogue-to-digital converter and software described above.

\section{Transcranial Magnetic Stimulation (TMS)}

TMS (MagPro Compact, MagVenture, Farum, Denmark) was used to either inhibit or activate the motor cortical area of the right plantar flexor muscles in the left hemisphere, slightly left of the vertex, via a double-cone coil (D-B80 Butterfly Coil, MagVenture, Farum, Denmark). The coil had monophasic current running through its centre in an anterior-posterior direction. The vertex was marked on the scalp and defined as halfway between the left and right processus zygomaticus ossis temporalis and halfway between the os nasale and the external occipital protuberance. The vertex location helped to find the optimal location for TMS of the motor cortical area innervating the right plantar flexor muscles (TMS hotspot), which is generally defined as the position in which a single stimulation evokes the largest peak-to-peak MEP amplitude in the target muscle (Siebner and Ziemann, 2007). In order to find the TMS hotspot, several stimuli were delivered while the TMS coil was slightly left, in front or behind the vertex, while participants performed fixed-end plantar flexion contractions at $30 \%$ of their maximal voluntary EMG activity (MVA) as measured during maximal voluntary fixed-end contractions (see experimental protocol). Once the TMS hotspot was located, the wings of the coil were marked on the scalp with a semi-permanent marker. Subthreshold and suprathreshold intensities were then determined by either decreasing or increasing the stimulator output. To achieve a suppression of soleus muscle activity, stimulator output was reduced in small increments until the active motor threshold (AMT) was reached. This threshold was defined as the point when 
206 stimulation during 30\% MVA resulted in visible MEP responses in only five of ten consecutive 207 trials (Petersen et al., 2001). Once the AMT was determined, stimulation intensity was slightly 208 reduced again so that MEPs were no longer elicited. For activation of the motor cortex, 209 stimulator output was increased until MEPs were clearly visible in comparison to the 210 immediately preceding background EMG in at least five consecutive trials.

211

212 Ultrasound imaging

213 Muscle fascicle behaviour of the MG from three participants was examined during 214 familiarisation sessions using B-mode ultrasound imaging (LS128 CEXT-1Z, Telemed, Vilnius, 215 Lithuania) to ensure muscle fascicle stretch occurred during ankle rotation in the stretch-hold 216 condition. Ultrasound images were recorded using a flat, linear, 128-element transducer 217 (LV7.5/60/128Z-2, $6 \mathrm{MHz}, 60 \times 50 \mathrm{~mm}$ (width x depth)) operating at $\sim 60 \mathrm{fps}$ in EchoWave II 218 software (Telemed, Vilnius, Lithuania). The transducer was placed on the skin over the MG mid219 muscle belly in the longitudinal plane and rotated to obtain a clear image with continuous 220 aponeuroses and muscle fascicles. The position of the probe was marked on the skin to ensure 221 consistent placement during the stretch-hold contractions.

222

223

224

225

226

227

228

229

230

231

232

233

234

235

236

237

238

239

240

241

242

243

244

245

\section{Contraction conditions}

The experiment involved participants performing submaximal plantar flexion contractions at $30 \%$ MVA. The background activity level was controlled throughout the contractions by having participants visually match their SOL EMG amplitude (moving 0.25 -s root-mean-square (RMS) amplitude calculation) to a horizontal cursor on a computer monitor. The conditions involved fixed-end reference contractions (REF) at $20^{\circ}$ dorsiflexion (DF) and active stretch-hold contractions (STR) from $0^{\circ}-20^{\circ} \mathrm{DF}\left(0^{\circ}\right.$ refers to the sole of the foot being perpendicular to the shank).

All contractions started with a 2-s linear ramp from 0-30\% MVA. Following the ramp, the isometric steady state during REF was maintained for 13-s. During STR, the ramp was followed by a $1-\mathrm{s}$ isometric steady state at the initial ankle joint angle $\left(0^{\circ} \mathrm{DF}\right)$, before the active plantar flexor muscles were stretched to an ankle joint angle of $20^{\circ} \mathrm{DF}$ at an angular velocity of $30^{\circ} \mathrm{s}^{-1}$. Following active stretch, participants maintained the subsequent isometric steady state for $\sim 11$-s, resulting in an overall contraction duration of 15-s (Fig. 1).

\section{Experimental protocol}

Participants attended two sessions on two different days. In the first session, participants were familiarised with the test protocol and trained to perform maximal voluntary fixed-end contractions (MVC) and the submaximal contractions described above. Additionally, participants were familiarised with TMS.

The second session consisted of the test protocol (Fig. 2). After a short warm-up (eight submaximal plantar flexion contractions with increasing torque), at least two MVCs were 
247 performed to determine $100 \%$ MVA. Participants were instructed to push their forefoot into the

248 footplate as hard as possible and to maintain the contractions so that a torque plateau was clearly

249 visible. MVC torque was calculated as the difference between peak torque during the contraction

250 and the mean baseline over $500 \mathrm{~ms}$ prior to the beginning of contraction. To ensure that the

251 MVCs were repeatable, peak-to-peak torques were required to be within a 5\% range. $100 \%$

252 MVA was determined as the smoothed (moving 0.25-s RMS amplitude calculation) SOL EMG

253 amplitude at peak MVC torque from the MVC with the highest peak-to-peak torque. Three

254 minutes of rest was provided between MVCs to minimise fatigue.

255

256

Following the MVCs, the TMS hotspot and the subthreshold and suprathreshold TMS intensities

257

258 were determined during sustained fixed-end contractions at 30\% MVA. Once hotspot and

259

260

261

262

263

264

265

266

267

268

269 stimulation intensities were determined, 100 subthreshold stimulations were delivered for each contraction condition to study motor cortex inhibition. For this purpose, contractions from each condition (i.e. STR or REF) were separated into ten sets with ten TMS $(1 \mathrm{~Hz})$ during the isometric steady state phase of the contractions at $20^{\circ} \mathrm{DF}$ starting 5 -s following contraction onset (Fig. 1). Contractions were randomised and a minimum of three minutes rest was provided between each set. Activation of the motor cortex was investigated by providing 20 suprathreshold stimulations per contraction condition. Suprathreshold stimulations were delivered at the same time points as the subthreshold stimulations, but participants performed only two contractions per condition. The contractions with suprathreshold TMS were always performed after all sets of the subthreshold stimulations had been completed. During all contractions, trials were excluded and repeated if participants could not maintain their soleus EMG activity within 25-35\% MVA.

270

271

272

\section{Data analysis}

\section{Residual Force Enhancement ( $\mathrm{rFE}$ )}

$273 \mathrm{rFE}$ for the subthreshold and suprathreshold conditions was determined by calculating the difference between the isometric steady-state torque during the STR and REF conditions. Net

275 plantar flexion torque was averaged across contractions for each contraction condition and mean

276

277 $\mathrm{rFE}$ was calculated during the isometric steady state at $20^{\circ} \mathrm{DF}$, from $500-990 \mathrm{~ms}$ after each TMS

278

279 stimulus. While most rFE studies have analysed torque or force with a $500 \mathrm{~ms}$ time window, we excluded the final $10 \mathrm{~ms}$ to ensure that the stimulations delivered at $1 \mathrm{~Hz}$ would not affect our analysis. Stimulations delivered before the isometric steady state of SOL muscle activity were excluded. In cases where participants showed no rFE for a specific stimulation condition (i.e. suprathreshold or subthreshold TMS), their data were excluded from statistical analysis..

282

283

\section{TMS inhibition}

284

285

Suppression of muscle activity (SOL, MG and LG) following subthreshold TMS was determined via averaged rectified raw EMG signals from each muscle and contraction condition. Waveform

286

287 averages were calculated over a time window of $100 \mathrm{~ms}$ after stimulation and again, stimulations delivered before the isometric steady state of SOL muscle activity were excluded. The latency 
288

289

290

291

292

293

294

295

296

297

298

299

300

301

302

303

304

305

306

307

308

309

310

311

312

313

314

315

316

317

318

319

320

321

322

323

324

325

326

327

328

and duration of EMG amplitude suppression were determined using the evaluation methods by Petersen et al. (2001) and Zuur et al. (2010). The onset of EMG amplitude suppression was marked when the EMG amplitude first dropped under the background EMG amplitude (mean value calculated 15-25 ms following stimulation) for at least $4 \mathrm{~ms}$. The offset of EMG amplitude suppression was calculated when the EMG amplitude rose above the background EMG amplitude for at least $1 \mathrm{~ms}$ (Zuur et al., 2010) (Fig. 3). The mean EMG amplitude between the onset and offset of suppression was calculated and compared with the background EMG amplitude right before suppression.

Torque production following TMS-induced EMG amplitude suppression was analysed via moving correlations (50-ms time intervals) between the torque of the STR and REF conditions. A reduction in torque due to TMS-induced EMG amplitude suppression was identified when torque data of REF and STR contractions were correlated ( $r \geq 0.7$, i.e. a very large effect). This data analysis was based on the random fluctuations in torque steadiness that should result in noncorrelated torque signals. However, when torque was affected by the TMS-induced EMG amplitude suppression in a similar manner, large positive correlations were expected. Once the time window of torque reduction was identified, the torque offset between the REF and STR conditions was removed by subtracting the mean torque difference between REF and STR over the first $10 \mathrm{~ms}$ after the onset of torque reduction. Finally, the mean torque during the period of TMS-induced torque reduction (i.e. during the times where $r \geq 0.7$ ) was calculated for REF and STR and compared between contraction conditions.

\section{TMS activation}

MEPs of SOL, MG and LG following suprathreshold TMS were calculated as peak-to-peak amplitudes from the raw EMG signals and averaged across contractions for each muscle and contraction condition (Lewis et al., 2014) (Fig. 4). Additionally, the silent period (SP) duration was analysed for both contraction conditions as the time from stimulation to the end of the SP. The end of the SP was defined as the time when the EMG signal following the MEP exceeded the threefold standard deviation (SD) of the raw EMG during the visually apparent SP (Fig. 4). SD during the SP was determined as the smallest SD of the raw EMG over a moving 30-ms window. The sizes of superimposed twitch torques following suprathreshold TMS were calculated separately as the difference in torque between the peak of the twitch and the torque at the time of stimulation and averaged for each contraction condition.

\section{Statistical analysis}

A two-way repeated-measures ANOVA was used to assess if the difference in triceps surae muscle activity between REF and STR conditions across the tested muscles were significantly different (contraction type $\times$ muscle). Due to missing values, two-way repeated-measures restricted maximum likelihood mixed-effects models were used to test for significant differences in EMG amplitude suppression (6 missing values), MEP amplitude (2 missing values) and SP duration (5 missing values) between REF and STR conditions across the tested muscles 
329 (contraction type $\times$ muscle). If a significant interaction was observed, Bonferroni post-hoc 330 comparisons were performed to determine which muscle significantly differed between REF and 331 STR conditions. Paired t-tests or Wilcoxon signed-rank tests, based on the normality of paired 332 differences as assessed by Shapiro-Wilk tests, were used to test for significant differences in 333 steady-state plantar flexion torque, torque reduction following subthreshold TMS, and 334 superimposed twitch torque following suprathreshold TMS between REF and STR conditions.

335 The alpha level was set at 0.05 and statistical analysis was performed using Prism 9 software 336 (GraphPad, USA). Values are presented as mean \pm SD in the text.

337

338

339

340

341

342

343

344

345

346

347

348

349

350

351

352

353

354

355

356

357

358

359

360

361

362

363

364

365

366

367

368

\section{Results}

Five and four participants were excluded from statistical analysis as they did not show $\mathrm{rFE}$ following the subthreshold $(\mathrm{n}=9)$ and suprathreshold $(\mathrm{n}=10)$ stimulations, respectively.

\section{Contraction conditions}

During both contraction conditions, participants managed to maintain a constant level of muscle activity ( $\sim 30 \%$ MVA). EMG of SOL, MG and LG did not significantly differ between STR and REF conditions $(P=0.504)$ during the isometric steady state of contractions with suprathreshold TMS. However, for subthreshold TMS, while EMG of SOL or LG was not significantly different between conditions (SOL: $P>0.999$, LG: $P>0.999$ ), EMG of MG was significantly higher in the REF compared with STR condition ( $P=0.001$; see Supplementary Fig. 1). Ultrasound imaging confirmed that the muscle-tendon unit stretch during the $20^{\circ}$ dynamometer rotation of the stretch-hold contraction resulted in muscle fascicle stretch of the MG at 30\% MVA ( $n=3)$.

\section{Contractions with subthreshold TMS}

The isometric steady-state net plantar flexion torque in the STR condition exceeded the timematched steady-state torque in the REF condition (STR: 105.9 $\pm 34.5 \mathrm{Nm}$; REF: $99.0 \pm 33.4 \mathrm{Nm}$, $P=0.004$ ), which resulted in mean $\mathrm{rFE}$ of $7.3 \pm 4.2 \%$ (Fig. 5A).

Subthreshold stimulation of the motor cortex led to significant $(P<0.001)$ EMG amplitude suppression for SOL, MG and LG without any significant difference in amplitude suppression $(P$ $=0.794$ ) between STR and REF conditions. The mean EMG amplitude suppression ranged between $19-25 \%$ of the background EMG amplitude, it occurred 41-46 ms after stimulation, and it lasted 10-15 ms in total (Table 1). After accounting for the torque offset between the REF and STR conditions, the net plantar flexor torque following EMG amplitude suppression did not significantly differ $(P=0.729)$ between STR and REF conditions.

\section{Contractions with suprathreshold TMS}

The isometric steady-state net plantar flexion torque in the STR condition exceeded the timematched steady-state torque in the REF condition (STR: $89.4 \pm 26.0 \mathrm{Nm}$; REF: $82.2 \pm 27.2 \mathrm{Nm}$, $P=0.002$ ), which resulted in mean $\mathrm{rFE}$ of $10.8 \pm 10.0 \%$ (Fig. 5A). For all muscles analysed (SOL, MG, LG), MEP amplitudes $(P=0.529)$ and SP durations $(P=0.609)$ following 
369

370

371

372

373

374

375

376

377

378

379

380

381

382

383

384

385

386

387

388

389

390

391

392

393

394

395

396

397

398

399

400

401

402

403

404

405

406

407

suprathreshold TMS did not significantly differ between STR and REF conditions (MEP: Fig. 5B; SP: Table 2). TMS-evoked superimposed twitches following suprathreshold stimulations were $9.1 \pm 3.5 \mathrm{Nm}$ (STR) and $8.6 \pm 3.5 \mathrm{Nm}$ (REF), and did not significantly differ between STR and REF conditions $(P=0.131$, Fig. $5 \mathrm{C})$.

\section{Discussion}

The aims of this study were to determine whether corticospinal excitability is modulated in the presence of $\mathrm{rFE}$ and to assess whether an inhibition of motor cortical neurons affects muscle activity and the increased torque production in the presence of $\mathrm{rFE}$ compared with fixed-end reference contractions without $\mathrm{rFE}$. To achieve this, we compared reductions in EMG activity and torque production following inhibition of motor cortical neurons, and MEPs, SPs and superimposed twitch torques following activation of motor cortical neurons between submaximal stretch-hold and fixed-end contractions.

Participants managed to keep muscle activity constant (30\% MVA) throughout the fixed-end reference contractions and during the isometric steady state of the stretch-hold contractions. As the observed differences in MG background EMG were rather small (2\% MVA), they are considered negligible regarding the interpretation of the results. Mean $\mathrm{rFE}$ magnitudes of $7 \%$ (subthreshold TMS) and 11\% (suprathreshold TMS) were observed during the steady state in STR compared with REF, which is in line with former studies (Oskouei \& Herzog, 2005; Pinniger \& Cresswell, 2007; Seiberl et al., 2013; Paternoster et al., 2016). Inhibition of motor cortical neurons by subthreshold TMS caused reductions in EMG activity and net plantar flexion torque, however the reductions did not differ significantly between contraction conditions. Similarly, MEP amplitudes, SP durations and superimposed twitch torque amplitudes evoked by suprathreshold TMS were not significantly different between STR and REF conditions.

Following subthreshold TMS, we found a suppression of triceps surae muscle activity by 19$24 \%$ relative to the background activity. This is similar to the $\sim 15 \%$ EMG amplitude suppression in SOL induced by subthreshold TMS during walking and jumping (Petersen et al., 2001; Zuur et al., 2010), but smaller compared with the 50\% suppression in hand and arm muscles during voluntary fixed-end contractions (Davey et al., 1994). Also, the latency and the duration of the observed EMG amplitude suppression was similar to values reported previously (Petersen et al., 2001; Zuur et al., 2010). Importantly, the TMS-induced EMG amplitude suppression showed no significant difference between STR and REF conditions, which also resulted in similar magnitudes of torque reduction for both contraction conditions. This supports our prediction that inhibiting motor cortical neurons by subthreshold TMS would not affect the STR and REF conditions differently. 
408 Our data also support our prediction that suprathreshold TMS does not elicit larger MEP 409 amplitudes and SP durations, or larger superimposed twitch torque amplitudes in the presence of $410 \mathrm{rFE}$ compared with fixed-end reference contractions (twitch amplitude of $\sim 9 \mathrm{Nm}$ for both 411 contraction conditions). The unchanged MEP amplitudes and SP durations indicate that 412 corticospinal excitability was unaltered in the presence of $\mathrm{rFE}$, which, based on a re-analysis of 413 the previously published data is in line with the earlier studies on corticospinal excitability in the

Interestingly, we found that activation of the motor cortex with a given suprathreshold stimulation intensity elicited comparable twitch torque amplitudes for both contraction conditions, despite steady-state torques following active stretch being enhanced because of rFE. We think that this finding further supports our interpretation of an unaltered corticospinal in the presence of rFE. This is because increased cortical excitability, but unchanged spinal excitability in the presence or rFE, as observed by Hahn et al. (2012), should lead to larger superimposed twitches, while unchanged cortical excitability, but reduced spinal excitability in the presence of rFE, as observed by Sypkes et al. (2018), should lead to smaller superimposed twitches. However, as neither of these findings were observed in the current study, we think that the overall excitability of the neuromuscular system was unchanged in the presence of rFE.

426

Finally, from a mechanical point of view, the unchanged superimposed twitches are in opposite

428

429

430

431

432

433

434

435

436

437

438

439

440

441

442

443

444

445

446

447

448

to what would be expected based on the work of Merton (1954), who showed that superimposed twitch size decreases as the pre-stimulus torque of the voluntary contraction increases. Accordingly, we interpret the equally-sized superimposed twitches following TMS in the presence of $\mathrm{rFE}$ as support for the idea that $\mathrm{rFE}$ is not due to a higher number of cross bridges following active stretch, but due to passive structural elements within the muscle that are engaged during active stretch (Edman, Elzinga \& Noble, 1978; Nishikawa, 2016; Herzog, 2018; Freundt \& Linke, 2019). The contribution of such passive structural elements to the increased force and torque production following stretch could also explain the observed reduction in EMG activity following stretch observed in force/torque-matched contractions (Oskouei \& Herzog, 2005; Seiberl et al., 2012).

\section{Limitations}

First, we only performed TMS of the motor cortex to assess corticospinal excitability, which does not allow us to distinguish between cortical and spinal aspects of corticospinal excitability. Accordingly, the unchanged MEP amplitudes and SP durations that we found in the presence of rFE compared with the reference contractions do not exclude potential cortical and/or spinal modulations in the presence of rFE. Second, we did not obtain M-waves to normalise MEPs. Although the earlier studies (Hahn et al. 2012; Sypkes et al. 2018) reported statistically unchanged $\mathrm{M}$-wave amplitudes in the presence of $\mathrm{rFE}$, theoretically, we might have missed potential changes in MEP amplitudes due to possible changes in M-wave amplitudes. Further, the similar superimposed twitches in the STR and REF conditions provide support that the 
449 overall excitability of the neuromuscular system was unchanged. Finally, we did not obtain TMS 450 input-output curves, which would reveal the relative sizes of the measured MEPs. However, 451 when setting up the individual stimulation intensities, we ensured that MEPs could still increase 452 with increasing stimulation intensity so that changes due to the contraction conditions would be 453 detectable.

454

\section{Conclusions}

456 In conclusion, we found that subthreshold and suprathreshold TMS of motor cortical neurons

457 affected muscle activity and torque production, but the mechanical and neural responses to TMS 458 did not differ between stretch-hold and fixed-end reference contractions. This is in line with our 459 predictions and suggests that corticospinal excitability remains unaltered in the presence of $\mathrm{rFE}$. 460 This further suggests that the enhanced torque production following active muscle stretch is not 461 due to changes in corticospinal excitability, but that rFE is likely caused by a stretch-induced 462 engagement of passive structural elements.

463

\section{Acknowledgements}

465 None.

466

467 


\section{References}

Abbott BC, Aubert XM. 1952. The force exerted by active striated muscle during and after change of length. The Journal of Physiology 117:77-86.

472

473

474

475

476

477

478

479

480

481

482

483

484

485

486

487

488

489

490

491

492

493

494

495

496

Altenburg TM, Ruiter CJ de, Verdijk PWL, van Mechelen W, Haan A de. 2008. Vastus lateralis surface and single motor unit EMG following submaximal shortening and lengthening contractions. Applied Physiology, Nutrition, and Metabolism 33 (6):1086-1095.

Brownstein CG, Ansdell P, Škarabot J, Howatson G, Goodall S, Thomas K. 2018. An optimal protocol for measurement of corticospinal excitability, short intracortical inhibition and intracortical facilitation in the rectus femoris. Journal of the Neurological Sciences 394:45-56.

Brunello E, Reconditi M, Elangovan R, Linari M, Sun YB, Narayanan T, Panine P, Piazzesi G, Irving M, Lombardi V. 2007. Skeletal muscle resists stretch by rapid binding of the second motor domain of myosin to actin. Proceedings of the National Academy of Science U S A 104 (50):2011420119.

Chapman N, Whitting J, Broadbent S, Crowley-McHattan Z, Meir R. 2018. Residual Force Enhancement in Humans: A Systematic Review. Journal of Applied Biomechanics:1-29.

Contento VS, Dalton BH, Power GA. 2019. The Inhibitory Tendon-Evoked Reflex Is Increased in the Torque-Enhanced State Following Active Lengthening Compared to a Purely Isometric Contraction. Brain Sciences 10 (1).

Davey NJ, Romaiguère P, Maskill DW, Ellaway PH. 1994. Suppression of voluntary motor activity revealed using transcranial magnetic stimulation of the motor cortex in man. The Journal of Physiology 477 (Pt 2):223-235.

Edman KA, Elzinga G, Noble MI. 1978. Enhancement of mechanical performance by stretch during tetanic contractions of vertebrate skeletal muscle fibres. Journal of Physiology 281:139-155.

Freundt JK, Linke WA. 2019. Titin as a force generating muscle protein under regulatory control. Journal of Applied Physiology 126:1474-1482.

Hahn D, Hoffman BW, Carroll TJ, Cresswell AG. 2012. Cortical and Spinal Excitability during and after Lengthening Contractions of the Human Plantar Flexor Muscles Performed with Maximal Voluntary Effort. PLoS One 7 (11):e49907. 
497

498

499

500

501

502

503

504

505

506

507

508

509

510

511

512

513

514

515

516

517

518

519

520

521

522

523

524

525

Hahn D, Seiberl W, Schmidt S, Schweizer K, Schwirtz A. 2010. Evidence of residual force enhancement for multi-joint leg extension. Journal of Biomechanics 43 (8): 1503-1508.

Herzog W. 2014. Mechanisms of enhanced force production in lengthening (eccentric) muscle contractions. Journal of Applied Physiology 116 (11):1407-1417.

Herzog W. 2018. The multiple roles of titin in muscle contraction and force production. Biophysical Reviews 10 (4):1187-1199.

Jakobi JM, Kuzyk SL, McNeil CJ, Dalton BH, Power GA. 2020. Motor unit contributions to activation reduction and torque steadiness following active lengthening: A study of residual torque enhancement. Journal of Neurophysiology.

Jones AA, Power GA, Herzog W. 2016. History dependence of the electromyogram: Implications for isometric steady-state EMG parameters following a lengthening or shortening contraction. Journal of Electrophysiological Kinesiology 27:30-38.

Joumaa V, Herzog W. 2013. Energy cost of force production is reduced after active stretch in skinned muscle fibres. Journal of Biomechanics 46 (6):1135-1139.

Julian FJ, Morgan DL. 1979. The effect on tension of non-uniform distribution of length changes applied to frog muscle fibres. Journal of Physiology-London 293:379-392.

Martin PG, Gandevia SC, Taylor JL. 2006. Output of human motoneuron pools to corticospinal inputs during voluntary contractions. Journal of Neurophysiology 95 (6):3512-3518.

Martin PG, Hudson AL, Gandevia SC, Taylor JL. 2009. Reproducible measurement of human motoneuron excitability with magnetic stimulation of the corticospinal tract. Journal of Neurophysiology 102 (1):606-613.

Mazara N, Hess AJ, Chen J, Power GA. 2018. Activation reduction following an eccentric contraction impairs torque steadiness in the isometric steady-state. Journal of Sport and Health Science 7 (3):310-317.

Merton PA. 1954. Voluntary strength and fatigue. Journal of Physiology 123 (3):553-564.

Nishikawa K. 2016. Eccentric contraction: Unraveling mechanisms of force enhancement and energy conservation. The Journal of Experimental Biology 219 (Pt 2):189-196.

Oskouei AE, Herzog W. 2005. Observations on force enhancement in submaximal voluntary contractions of human adductor pollicis muscle. Journal of Applied Physiology 98 (6):2087-2095. 
526

527

528

529

530

531

532

533

534

535

536

537

538

539

540

541

542

543

544

545

546

547

548

549

550

551

552

553

554

555

556

Paquin J, Power GA. 2018. History dependence of the EMG-torque relationship. Journal of Electrophysiological Kinesiology 41:109-115.

Paternoster FK, Seiberl W, Hahn D, Schwirtz A. 2016. Residual force enhancement during multi-joint leg extensions at joint- angle configurations close to natural human motion. Journal of Biomechanics 49 (5): $773-779$.

Petersen NT, Butler JE, Marchand-Pauvert V, Fisher R, Ledebt A, Pyndt HS, Hansen NL, Nielsen JB. 2001. Suppression of EMG activity by transcranial magnetic stimulation in human subjects during walking. The Journal of Physiology 537 (2):651-656.

Pinniger GJ, Cresswell AG. 2007. Residual force enhancement after lengthening is present during submaximal plantar flexion and dorsiflexion actions in humans. Journal of Applied Physiology 102 (1): 18-25.

Raiteri BJ, Cresswell AG, Lichtwark GA. 2015. Ultrasound reveals negligible cocontraction during isometric plantar flexion and dorsiflexion despite the presence of antagonist electromyographic activity. Journal of Applied Physiology 118 (10):1193-1199.

Rassier DE. 2012. The mechanisms of the residual force enhancement after stretch of skeletal muscle: non-uniformity in half-sarcomeres and stiffness of titin. Proceedings of the Biological Society 279 (1739):2705-2713.

\section{Rossini PM, Burke D, Chen R, Cohen LG, Daskalakis Z, Di Iorio R, Di Lazzaro V, Ferreri F,} Fitzgerald PB, George MS, Hallett M, Lefaucheur JP, Langguth B, Matsumoto H, Miniussi C, Nitsche MA, Pascual-Leone A, Paulus W, Rossi S, Rothwell JC, Siebner HR, Ugawa Y, Walsh V, Ziemann U. 2015. Non-invasive electrical and magnetic stimulation of the brain, spinal cord, roots and peripheral nerves: Basic principles and procedures for routine clinical and research application. An updated report from an I.F.C.N. Committee. Clinical Neurophysiology 126 (6):1071-1107.

Rothwell J. 1997. Techniques and mechanisms of action of transcranial stimulation of the human motor cortex. Journal of Neuroscience Methods 74 (2):113-122.

Seiberl W, Hahn D, Herzog W, Schwirtz A. 2012. Feedback controlled force enhancement and activation reduction of voluntarily activated quadriceps femoris during sub-maximal muscle action. Journal of Electromyography and Kinesiology 22 (1):117-123.

Seiberl W, Hahn D, Kreuzpointner F, Schwirtz A, Gastmann U. 2010. Force Enhancement of Quadriceps Femoris in Vivo and Its Dependence on Stretch-Induced Muscle Architectural Changes. Journal of Applied Biomechanics (26):256-264. 
557 Seiberl W, Paternoster F, Achatz F, Schwirtz A, Hahn D. 2013. On the relevance of residual force 558 enhancement for everyday human movement. Journal of Biomechanics 46:1996-2001.

559 Seiberl W, Power GA, Hahn D. 2015. Residual force enhancement in humans: Current evidence and 560 unresolved issues. Journal of Electromyography and Kinesiology 25 (4):571-580.

561 Sypkes CT, Kozlowski BJ, Grant J, Bent LR, McNeil CJ, Power GA. 2018. The influence of residual 562 force enhancement on spinal and supraspinal excitability. PeerJ 6:e5421.

563 Zuur AT, Lundbye-Jensen J, Leukel C, Taube W, Grey MJ, Gollhofer A, Nielsen JB, Gruber M.

564 2010. Contribution of afferent feedback and descending drive to human hopping. The Journal of

565 Physiology 588 (Pt 5):799-807.

566 


\section{Table $\mathbf{1}$ (on next page)}

EMG responses following subthreshold transcranial magnetic stimulation.

Mean \pm SD values of EMG amplitude suppression following subthreshold transcranial magnetic stimulation from soleus (SOL), medial gastrocnemius (MG) and lateral gastrocnemius (LG) muscles during stretch-hold (STR) and fixed-end reference (REF) contractions. 


\begin{tabular}{llll} 
& SOL & MG & LG \\
\hline EMG suppression REF [\%] & $24.0 \pm 6.7$ & $24.7 \pm 7.2$ & $19.4 \pm 2.3$ \\
EMG suppression STR [\%] & $24.2 \pm 5.4$ & $23.7 \pm 6.9$ & $20.9 \pm 6.4$ \\
Latency REF [ms] & $44 \pm 4$ & $41 \pm 5$ & $44 \pm 5$ \\
Latency STR [ms] & $45 \pm 3$ & $43 \pm 6$ & $46 \pm 5$ \\
Duration REF [ms] & $14 \pm 4$ & $15 \pm 4$ & $12 \pm 5$ \\
Duration STR [ms] & $13 \pm 6$ & $12 \pm 4$ & $10 \pm 4$
\end{tabular}

1

2 


\section{Table 2 (on next page)}

EMG responses following suprathreshold transcranial magnetic stimulation.

Mean \pm SD values of motor evoked potentials (MEPs) and silent periods (SPs) after suprathreshold transcranial magnetic stimulation from soleus (SOL), medial gastrocnemius (MG) and lateral gastrocnemius (LG) muscles during stretch-hold (STR) and fixed-end reference (REF) contractions. 


\begin{tabular}{llll} 
& SOL & MG & LG \\
\hline MEPs REF [V] & $1.30 \pm 0.78$ & $2.54 \pm 2.67$ & $1.19 \pm 0.46$ \\
MEPs STR [V] & $1.37 \pm 0.72$ & $2.70 \pm 2.97$ & $1.14 \pm 0.50$ \\
SPs REF [ms] & $115 \pm 31$ & $112 \pm 22$ & $118 \pm 29$ \\
SPs STR [ms] & $116 \pm 31$ & $115 \pm 23$ & $123 \pm 34$
\end{tabular}

1

2

3 
Figure 1

Example data from the stretch-hold (STR, blue) and fixed-end reference (REF, grey) contraction conditions.

A: The traces show soleus (SOL) EMG (moving 0.25-s root-mean-square (RMS) amplitude calculations). Transcranial magnetic stimulation (TMS, vertical black lines) was delivered at 1 $\mathrm{Hz}$ from $5 \mathrm{~s}$ after contraction onset (marked as time zero). In case the first stimulation was delivered before SOL EMG reached the target level, the stimulation was excluded from analysis. B: Traces show the corresponding crank arm angles.

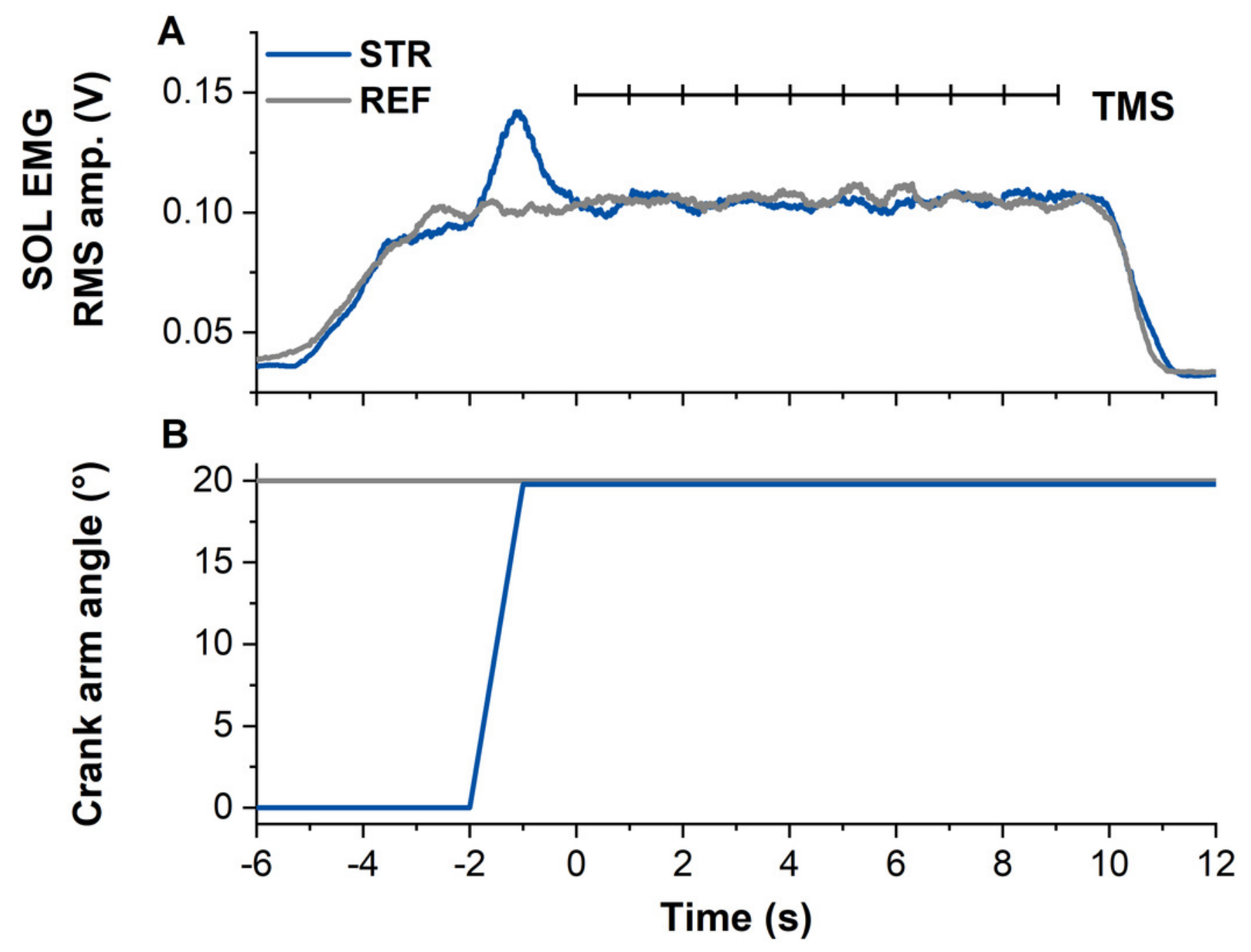




\section{Figure 2}

Schematic diagram of the experimental protocol.

A: The protocol started and ended with maximal voluntary contractions (MVC, dark grey). Transcranial magnetic stimulation (TMS) hotspot and sub-/suprathreshold intensities (light grey) were then determined before data collection. Twenty contractions with subthreshold TMS (dark blue) and 4 contractions with suprathreshold TMS (light blue) were separated into three sets, with 5 min rest between each set. B: Schematic description of contractions within sets (blue bars) and delivery of TMS (same procedure for sub- and suprathreshold TMS). Vertical black lines indicate the timing of TMS during the submaximal contractions. Each set during subthreshold TMS consisted of 10 contractions of $15 \mathrm{~s}$ duration, followed by a $3 \mathrm{~min}$ rest. 
A

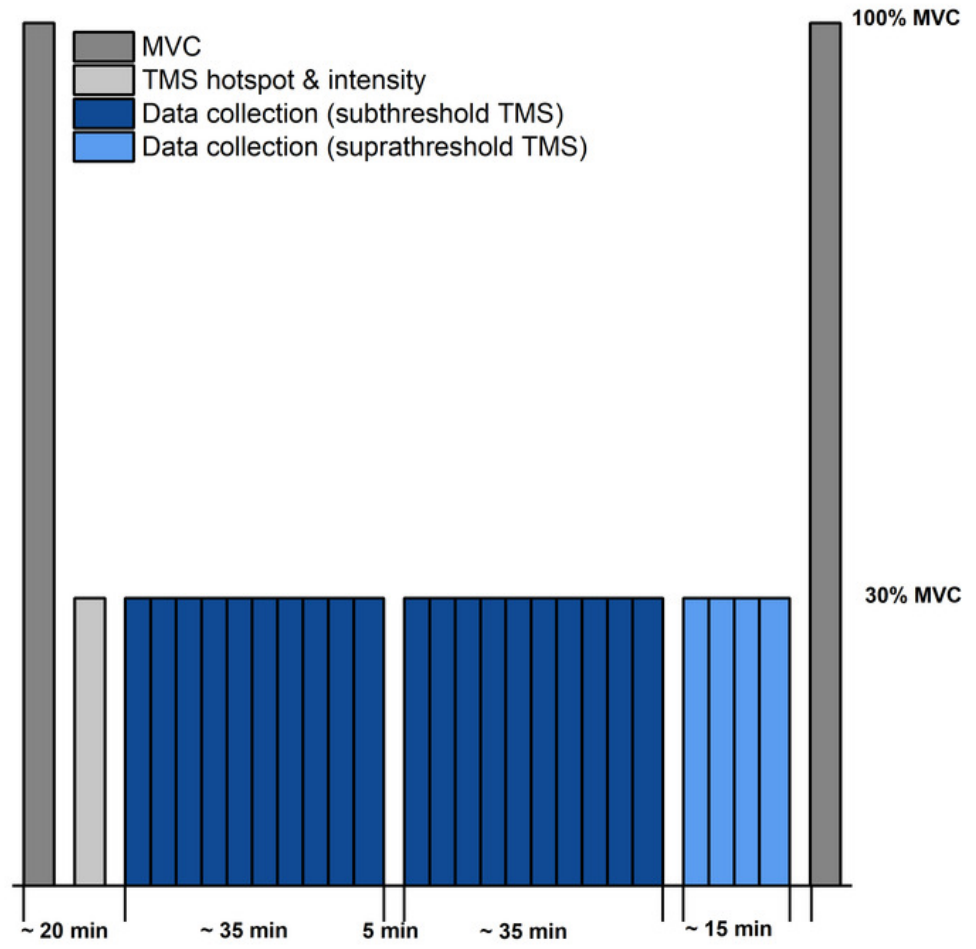

B TMS TMS
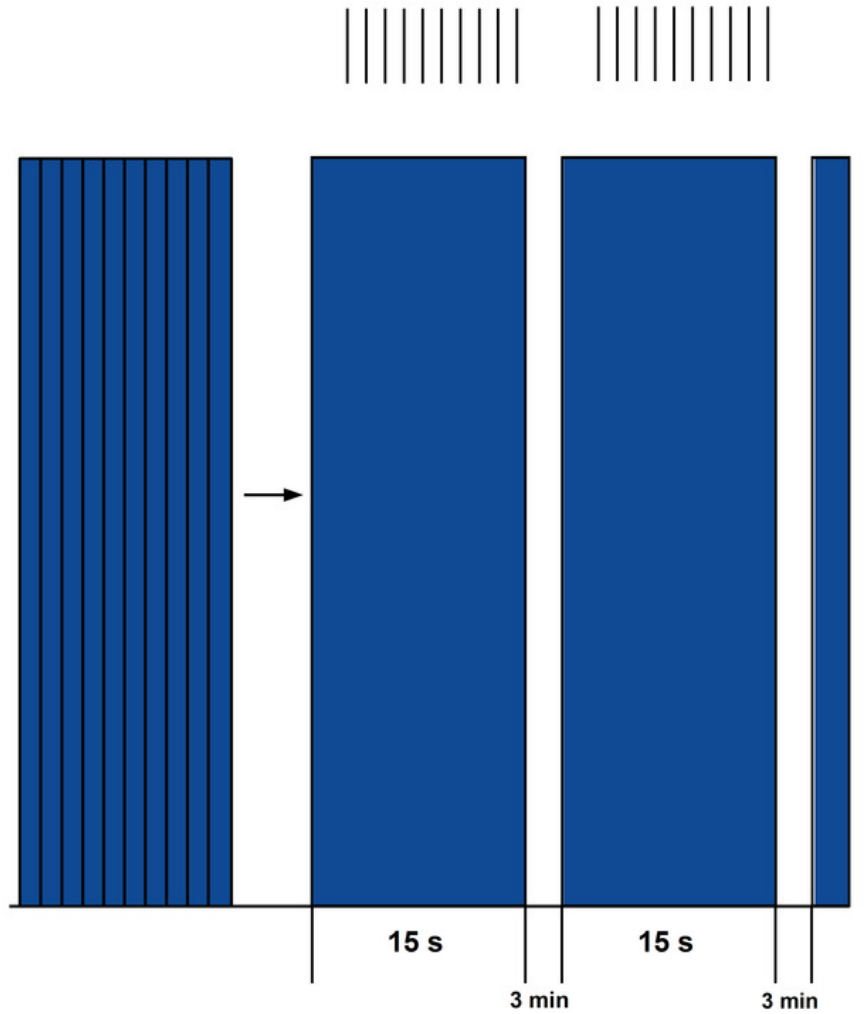
Figure 3

Example data of rectified and averaged soleus (SOL) EMG from contractions with subthreshold transcranial magnetic stimulation (TMS).

The horizontal dotted line represents the averaged background EMG amplitude calculated from 15-25 ms following stimulation. Vertical dashed lines indicate onset and offset of EMG amplitude suppression following TMS. Time zero indicates the time of stimulation.

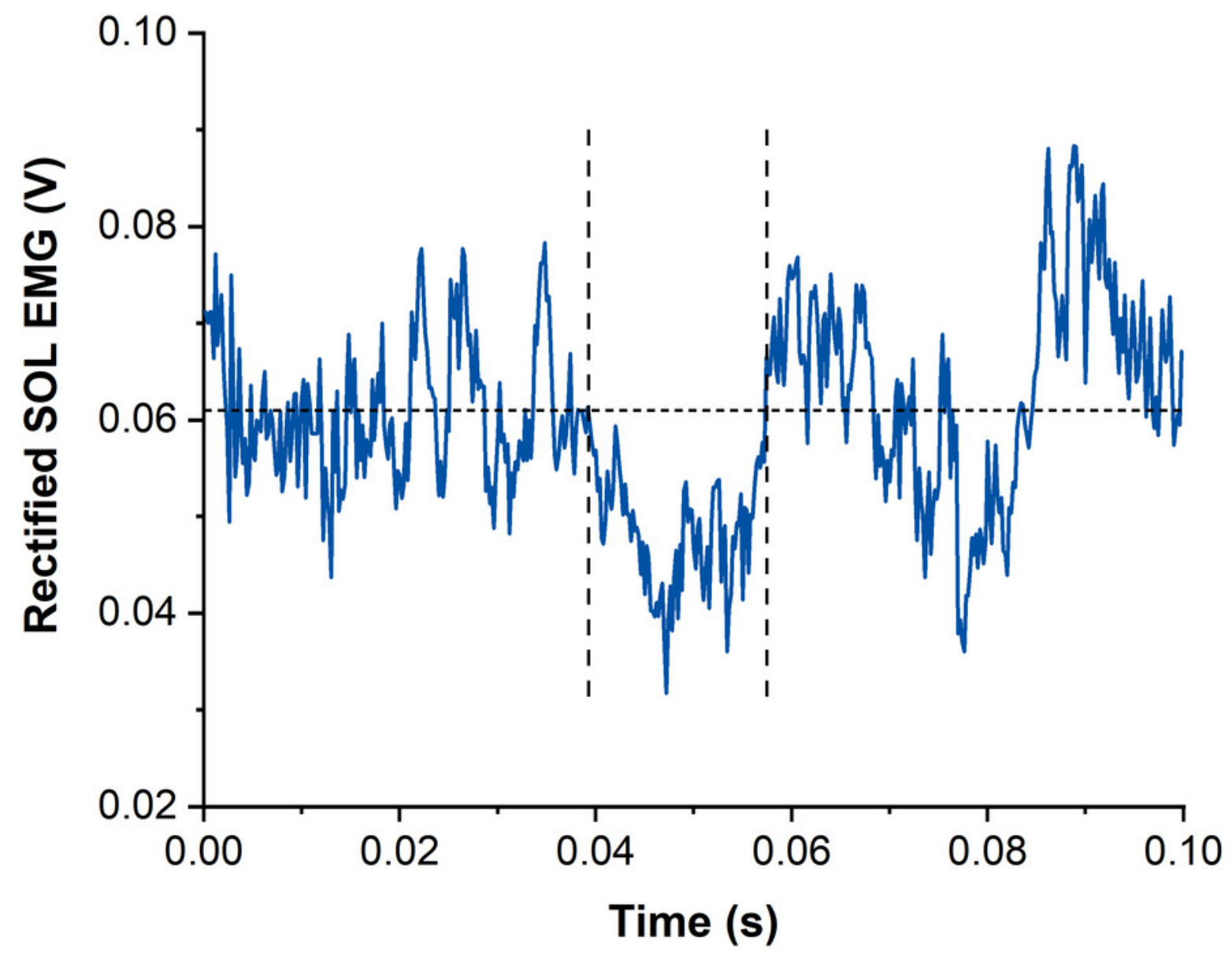


Figure 4

Representative example of motor evoked potentials (MEPs) and silent periods (SPs) from raw soleus (SOL) EMG signals elicited by suprathreshold transcranial magnetic stimulation (TMS).

The light grey traces show the single MEPs and the dark blue trace shows the average of all SOL MEPs from one participant. The horizontal dashed black line indicates the duration of the silent period (SP) and the vertical black line indicates the peak-to-peak amplitude of the largest single MEP. Time zero indicates the time of stimulation.

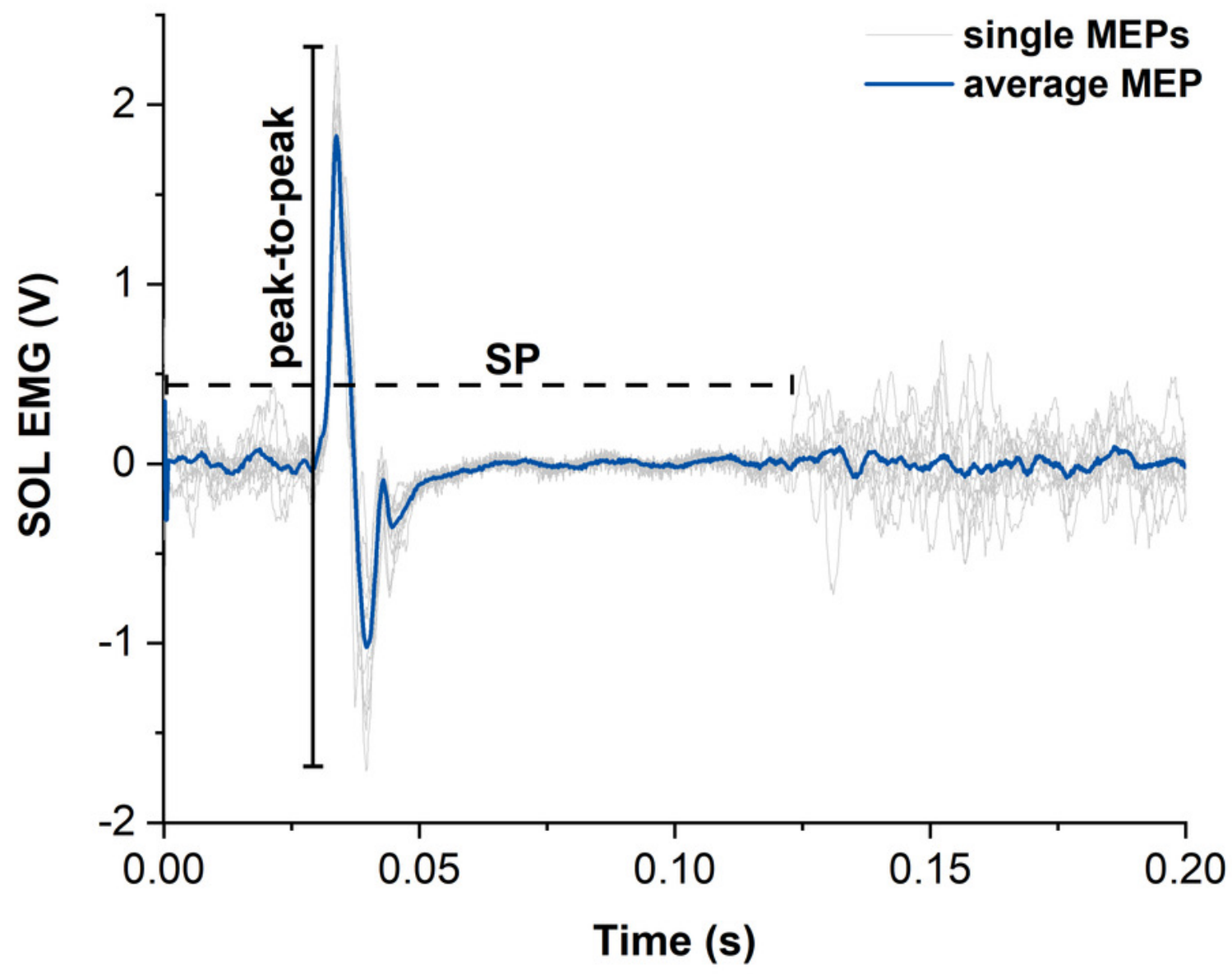




\section{Figure 5}

Residual force enhancement (rFE), motor evoked potentials (MEPs), and twitch torques.

A: Residual force enhancement during stretch-hold (STR) contractions normalized to the time-matched torque during fixed-end reference (REF) contractions. The open circles and error bars represent the means and $95 \%$ confident intervals for the contractions with subthreshold transcranial magnetic stimulation (TMS) (left) and suprathreshold TMS (right), respectively. The grey dots represent the individual data points. B: Normalised MEP amplitude differences between STR and REF. The open circles and error bars represent the means and the $95 \%$ confident intervals and the grey dots represent the individual data points for soleus (SOL), medial gastrocnemius (MG) and lateral gastrocnemius (LG) muscles, respectively. C: Superimposed twitch torques after suprathreshold TMS. The open circles and error bars represent the means and the $95 \%$ confident intervals and the grey dots represent the individual data points for REF (left) and STR (right). 

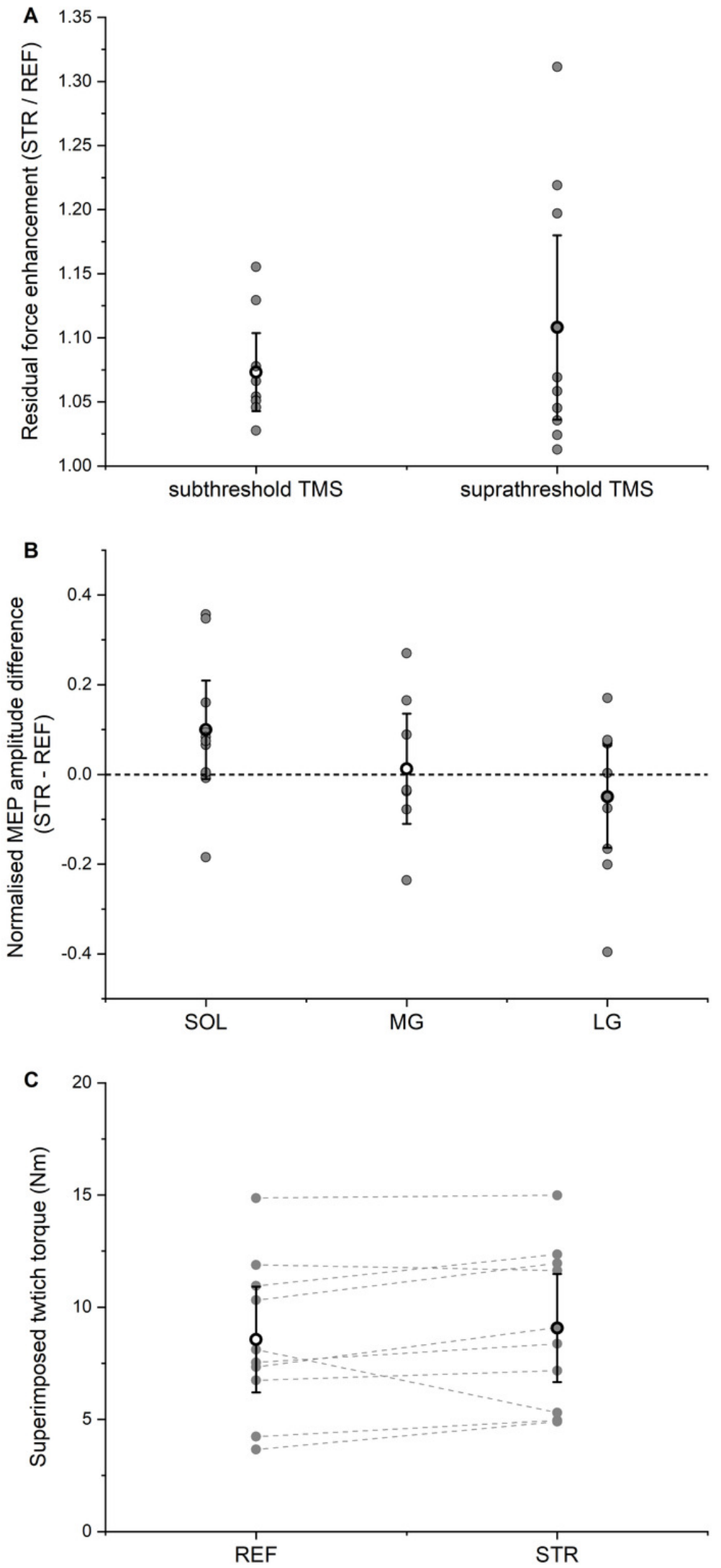